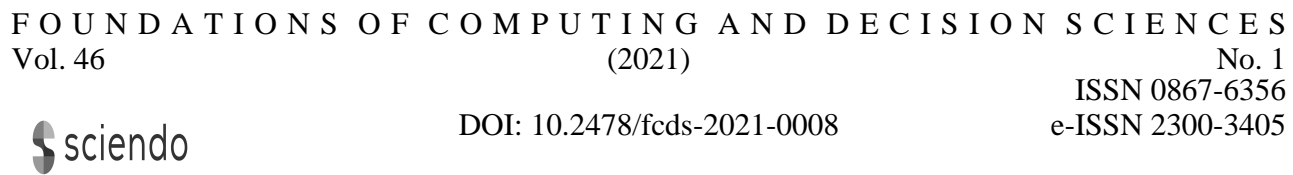

\title{
Understanding the Impact of COVID-19 on Global Financial Network Using Graph Based Algorithm: Minimum Spanning Tree Approach
}

\author{
Veysel Fuat Hatipoğlu *
}

\begin{abstract}
In this paper effects of COVID-19 pandemic on stock market network are analyzed by an application of operational research with a mathematical approach. For this purpose two minimum spanning trees for each time period namely before and during COVID-19 pandemic are constructed. Dynamic time warping algorithm is used to measure the similarity between each time series of the investigated stock markets. Then, clusters of investigated stock markets are constructed. Numerical values of the topology evaluation for each cluster and time period is computed.
\end{abstract}

Keywords: Big data, big data analysis, minimum spanning tree, COVID-19 pandemic, dynamic time warping, stock market

\section{Introduction}

Operations research which is a military science oriented discipline, involves characteristics of mathematics and statistics. Operations research has several successful applications in several other disciplines. Some of them are as follows: Accounting, supply chain management, economics, finance, business strategy, transportation, communication, logistics and sports. In this paper, we deal with an application of operations research to economics. In particular, we apply minimum spanning tree approach to stock markets based on dynamic time warping algorithm as a distance metric. Dynamic time warping algorithm is valuable due to its advantages of analyzing asynchronous data regardless Dynamic time warping algorithm also enables us to analyze the data with different length of time series. Minimum spanning tree approach based on dynamic time warping algorithm is relatively new subject and applied to various sciences. In [35] authors applied in medicine. The authors described the interactions

*Fethiye Faculty of Business Administration, Muğla Sıtkı Koçman University, Muğla, Turkey, veyselfuat.hatipoglu@mu.edu.tr 
between distributed brain regions by establishing a minimum spanning tree based on dynamic time warping algorithm. In order to understand the behavior of the objects, minimum spanning tree algorithm with dynamic time warping algorithm has been studied in [29] by clustering the motion patterns. In [21] authors constructed a minimum spanning tree from the data of Vietnamese stock market between 2011-2012. During that period there was a financial crisis in Vietnam. According to authors findings, results are similar comparing to those of world-wide financial crisis 2008. Another recent application of minimum spanning tree to stock exchanges is [13] by obtaining a sectorial hierarchy among some sectors in Turkish stock market BIST100. We refer the interested reader to $[16,18,22]$ for detailed analysis on financial markets by using minimum spanning tre technique.

COVID-19 pandemic has been started on 31 December 2019 according to official records. That disease which has been officially announced as pandemic by WHO as of 11 March 2020 does not affect only the public health and daily habits but also financial and business life of the countries. According to official records as of end of May 2020 over 6 million people have been transmitted and caused more than 350 thousand deaths in less than five months all over the world. Governments of several countries decided to take some actions such as quarantines, regulations for working hours in some sectors, lockdowns, stay at home regulations and social distancing rules. Number of the studies focusing on the effects of COVID-19 pandemic is increasing day by day in various fields of science. Some papers counts COVID-19 pandemic as the biggest public health problem of this century [10,24]. Several articles published in various fields of medical sciences related to origin and spread of virus, cure and vaccination strategies for the disease, and epidemiological models based on COVID$19[26,12,9,14,19]$. The socio-economic impacts of COVID-19 are reviewed in [23]. In $[25,28]$ different forecasting results for the spread of coronavirus are obtained. Moreover the environmental effects of COVID-19 are widely discussed in [30] and the references there in.

COVID-19 pandemic has important consequences on the financial markets. Some economic and social impact of COVID-19 pandemic can be found in In $[11,4,34]$. Several authors investigated the economical impact of COVID-19 particularly on stock markets. Here we summarize some information on some of them. In [20] authors modelled stock price variation during the coronavirus spread around the world by sampling 10 stock market indices (S\&P 500 (United States), FTSE 100 (United Kingdom), NIKKEI 225 (Japan), CAC 40 (France), NIFTY 50 (India), S\&P/TSX Composite (Canada), DAX (Germany), SMI (Switzerland), KOSPI (Korea), and All Ordinaries (Australia)). In [3], authors use panel data analysis to test the impact of the COVID-19 pandemic on the Chinese stock market. Regarding to the authors results, it is found that both the daily growth in total cases of death caused by COVID-19 and the daily growth in total confirmed cases have negative effects on stock returns significantly among all companies. The authors of [6] studied on the US stock market S\&P 500 and indicated the potential social and political reasons for the unpredicted stock market response to the COVID-19 pandemic. Social and political expectations for post-COVID-19 can be figured out by investigating the behavior of stock markets as in [32]. In [31], the authors examined the effect of COVID-19 on 
26 emerging stock markets. The stock market indices are defined as a function of oil price shocks, exchange rates, and infection rate. According to the authors results it is seen that the Asian emerging markets are the most effected ones. In [5], the authors examine the response of the stock markets to COVID-19 pandemic by using daily data of stock market returns obtained from 64 countries, COVID-19 daily cases and deaths. In both studies $[31,5]$ the authors used synchronous and daily data.

As a result of such regulations economies and business life of countries directly and indirectly affected in several sectors such as utilities, technology, industrials, energy, chemicals, banking and financing. Due to the spread of virus each country started to affected by the virus on different dates. Therefore correlating the financial markets between countries is impossible with classical correlation distances. In such cases dynamic time warping algorithm is a powerful tool to analyze the interaction among the global financial market network.

Differing from previous studies $[31,5,27,1,2,7,8]$, in this article, we consider the speed of change of the daily returns of the stock markets as a variable by using dynamic time warping algorithm and we analyze the behavior of interaction between stock markets before and during the economical crisis caused by COVID-19 pandemic. For this purpose we build up a hierarchy among the stock exchanges for a limited time period of before and during the virus effect. Then we compare both results. Moreover topology evolution of the obtained clusters are presented.

\section{Methodology and Data}

Research is conducted on 23 stock markets covering two time frames. The first time frame starts from January 01, 2018 ends at the date of first reported COVID-19 case in the origin country of the stock exchange. The second time frame is from the date of first reported COVID-19 case in the origin country of the stock exchange till May 27, 2020. The daily value changes of stock exchanges are investigated. For all computations Mathematica 11 software is used. Countries and reported first COVID19 case chronology is given by Table 1 and the studied stock markets, countries and data periods are listed in Table 2 .

Classical correlation coefficients are widely used to determine the similarity distance between the nodes of graphs, but calculation of classical correlation coefficients need synchronous data. Unfortunately the studied data is asynchronous because of the different dates for the first reported cases of COVID-19 in each country. To analyze such data, dynamic time warping would be a useful tool. Dynamic time warping algorithm, uses the two temporal sequences of a logarithmic return of the daily closure prices of each stock exchange during studied time period. So by using dynamic time warping algorithm given in [33], we compute the distance matrix $D$ to measure the similarity between each pair of stock exchanges. Then computed distance matrix is represented by minimum spanning trees in Figure 1 and Figure 2 for both time frames. By this way, we may analyze the interactions among the asynchronous time evolution of each pair of stock exchanges. Determining clusters from the minimum spanning tree of investigated stock markets is not easy. Therefore we build dendrograms for 
Table 1. List of countries and the date of first reported COVID-19 case

\section{Country}

Argentina

Brazil

China

Germany

India

Japan

Mexico

Nigeria

Russia

Spain

Turkey

United States

of America

\section{First reported COVID-19 case}

March 03, 2020
February 25, 2020
December 01, 2019
January 27, 2020
January 30, 2020
January 16, 2020
February 28, 2020
February 27, 2020
January 31, 2020
January 31, 2020
March 10, 2020

Country

Australia

Canada

France

Hong Kong

Italy

Malaysia

Netherlands

Poland

South Korea

Switzerland

United Kingdom
First reported COVID-19 case

January 01, 2020

January 25, 2020

January 24, 2020

January 22, 2020

January 30, 2020

January 25, 2020

February 27, 2020

March 04, 2020

January 20, 2020

February 27, 2020

January 31, 2020

January 20, 2020

Table 2. List of stock markets, symbols, related countries and investigated time periods

Stock market
S\&P Merval (MERV)
S\&P/ASX 200 (AXJO)
Bovespa (BVSP)
S\&P/TSX (GSPTSE)
Shanghai (SSEC)
CAC 40 (FCHI)
DAX (GDAXI)
FTSE China 50
(FTXIN25)
BSE Sensex 30 (BSESN)
FTSE Italia (FTITLMS)
Nikkei 225 (N225)
FTSE Malaysia
KLCI (KLSE)
S\&P/BMV IPC (MXX)
AEX (AEX)
NSE 30 (NGSE30)
WIG (WIG)
MOEX Russia (IMOEX)
KOSPI (KS11)
IBEX 35 (IBEX)
SMI (SSMI)
BIST 100 (XU100)
FTSE 100 (FTSE)
S\&P 500 (SPX)

Country
Argentina
Australia
Brazil
Canada
China
France
Germany
Hong Kong
India
Italy
Japan
Malaysia
Mexico
Netherlands
Nigeria
Poland
Russia
South Korea
Spain
Switzerland
Turkey
UK
USA

Time frame I

$01.02 .2018-02.03 .2020$

$01.02 .2018-01.24 .2020$

$01.02 .2018-02.21 .2020$

$01.02 .2018-01.24 .2020$

$01.02 .2018-11.29 .2020$

$01.02 .2018-01.23 .2020$

$01.02 .2018-01.24 .2020$

$01.02 .2018-01.21 .2020$

$01.01 .2018-01.29 .2020$

$01.02 .2018-01.29 .2020$

$01.04 .2018-01.15 .2020$

$01.02 .2018-01.24 .2020$

$01.02 .2018-02.27 .2020$

$01.02 .2018-02.26 .2020$

$01.02 .2018-02.26 .2020$

$01.02 .2018-03.03 .2020$

$01.03 .2018-01.30 .2020$

$01.02 .2018-01.17 .2020$

$01.02 .2018-01.30 .2020$

$01.03 .2018-02.24 .2020$

$01.02 .2018-03.03 .2020$

$01.02 .2018-01.30 .2020$

$01.02 .2018-01.17 .2020$
Time frame II

03.03.2020 - 05.27.2020

$01.28 .2020-05.27 .2020$

$02.26 .2020-05.27 .2020$

$01.28 .2020-05.27 .2020$

$12.02 .2020-05.27 .2020$

$01.24 .2020-05.27 .2020$

$01.27 .2020-05.27 .2020$

$01.22 .2020-05.27 .2020$

$01.30 .2020-05.27 .2020$

$01.30 .2020-05.27 .2020$

$01.16 .2020-05.27 .2020$

$01.27 .2020-05.27 .2020$ $02.28 .2020-05.27 .2020$ $02.27 .2020-05.27 .2020$ $02.27 .2020-05.27 .2020$ $04.03 .2020-05.27 .2020$ $01.31 .2020-05.27 .2020$ $01.20 .2020-05.27 .2020$ $01.31 .2020-05.27 .2020$ $02.27 .2020-05.27 .2020$ $04.03 .2020-05.27 .2020$ $01.31 .2020-05.27 .2020$ $01.21 .2020-05.27 .2020$ 
both time frames by using hierarchical clustering depending on dynamic time warping. The dendrograms are presented in Figure 3 and Figure 4. With respect to the first dendrogram, 21 investigated stock exchanges are separated into three clusters which are containing at least 4 stock markets. Other two stock markets namely BOVESPA (Brazil) and BIST100 (Turkey) are grouping as a couple omitted due to not having enough similarity distance with at least 3 other stock exchanges. Similarly with respect to the second dendrogram, 3 clusters with at least 4 stock exchanges formed by 21 stock exchanges for topology evolution of clusters. Hence two stock markets are omitted. Once the clusters are obtained, the sub minimum spanning trees for each cluster are also considered to analyze the topology evaluation of each cluster for both time frames. To analyze the topology evolution of considered clusters of stock exchanges, the evaluation criteria of [33] is applied. The mean similarity measure (MSM) for the $n \times n$ similarity matrix $D$ is defined as

$$
L_{M S M}=\frac{2}{n(n-1)} \sum_{i=1}^{n-1} \sum_{j=i+1}^{n} D_{i j}
$$

where $n$ is the number of stock exchanges in the investigated cluster. The normalized tree length (NTL) is given by [15] as,

$$
L_{N T L}=\frac{1}{(n-1)} \sum_{D_{i j} \in \Omega} D_{i j},
$$

where $\Omega$ is the set of edges, and $n-1$ is the number of edges in the considered minimum spanning tree. The characteristic path length (CPL) is defined in [17], in order to quantify the average minimal route between pairs of nodes as,

$$
L_{C P L}=\frac{1}{n(n-1)} \sum_{i, j: i \neq j} l_{i j},
$$

where $l_{i j}$ is the number of edges in the shortest path between nodes $i$ and $j$. By finding CPL compactness of the structure of investigated network might be understood. $L_{M S M}, L_{N T L}$ and $L_{C P L}$ values for the determined three clusters for each time frame are presented in Table 4 and Table 5 .

\section{Numerical Computation and Main Results}

In this section firstly two minimum spanning trees are constructed to demonstrate the interaction level between 23 stock markets before and during the economical crisis caused by COVID-19 pandemic. Both spanning trees are presented in Figure 1 and Figure 2. After constructing minimum spanning trees a dendrogram is plotted in order to visualize a hierarchical clusters. According to dendrogram in Figure 3 and Figure 4 we recognize a hierarchy among the stock exchanges and determine the groups. Then topology evaluation analysis is done for the obtained clusters for both time frames. Results are presented in Table 4 and Table 5. 


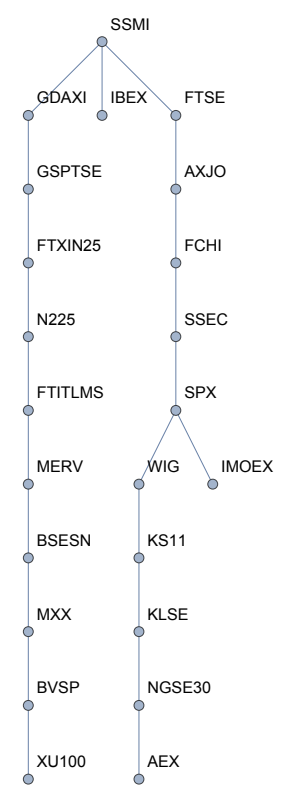

Figure 1. Minimum spanning tree of investigated stock markets before COVID-19

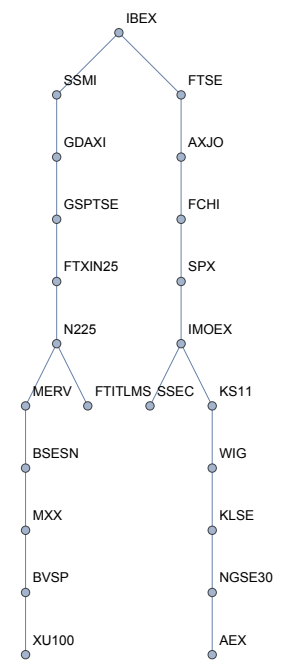

Figure 2. Minimum spanning tree of investigated stock markets during COVID-19 
According to minimum spanning tree in Figure 1 that represents the interaction between stock markets before COVID-19 pandemic, SMI (Switzerland) and S\&P 500 (USA) are the most affecting indices with three edges each. SMI directly affects DAX (Germany), IBEX 35 (Spain) and FTSE 100 (UK). S\&P 500 has an direct impact on Shanghai (China), WIG (Poland) and MOEX Russia (Russia) from view point of financial moves. On the other hand after the date of first announced case of each country for COVID-19 pandemic, interaction between financial markets has different main actors according to minimum spanning tree in Figure 2. Those indices are Nikkei 225 (Japan) and MOEX Russia with three edges each. It is seen that Nikkei 225 affects S\&P Merval (Argentina), FTSE China 50 (Hong Kong) and FTSE Italia (Italy), and MOEX Russia affects Shanghai, KOSPI (South Korea) and S\&P 500 more than the others. From Figure 1 and Figure 2 it is impossible to determine clusters among stock markets. Therefore dendrograms for both cases are plotted in Figure 3 and Figure 4. Moreover, topology evaluation for both situations are compared in Table 3. The average distance of edges in minimum spanning trees could be calculated by normalized tree length. From the decrease in normalized tree length in Table 3 one can see that during COVID-19 pandemic investigated stock markets get close to each other in the manner of similar behavior.

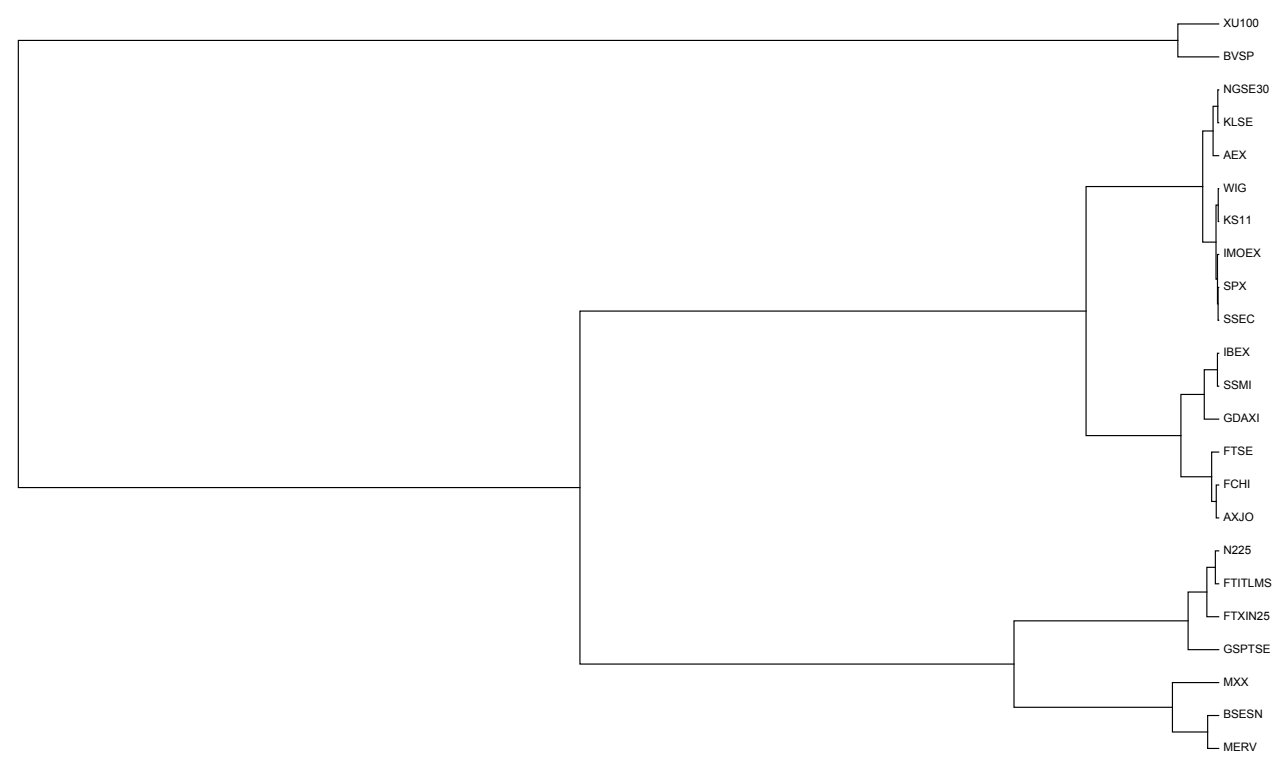

Figure 3. Minimum spanning tree of investigated stock markets before COVID-19

Three clusters are obtained from dendrogram in Figure 3. NSE 30 (Nigeria), FTSE Malaysia KLCI (Malaysia), AEX (Netherlands), WIG, KOSPI, MOEX Russia, S\&P 500 and Shanghai form first group. IBEX 35, SMI, DAX, FTSE 100, CAC 40 (France) and S\&P/ASX 200 (Australia) form second group. The third group is formed by the following indices, Nikkei 225, FTSE Italia, FTSE China 50, S\&P/TSX (Canada), 


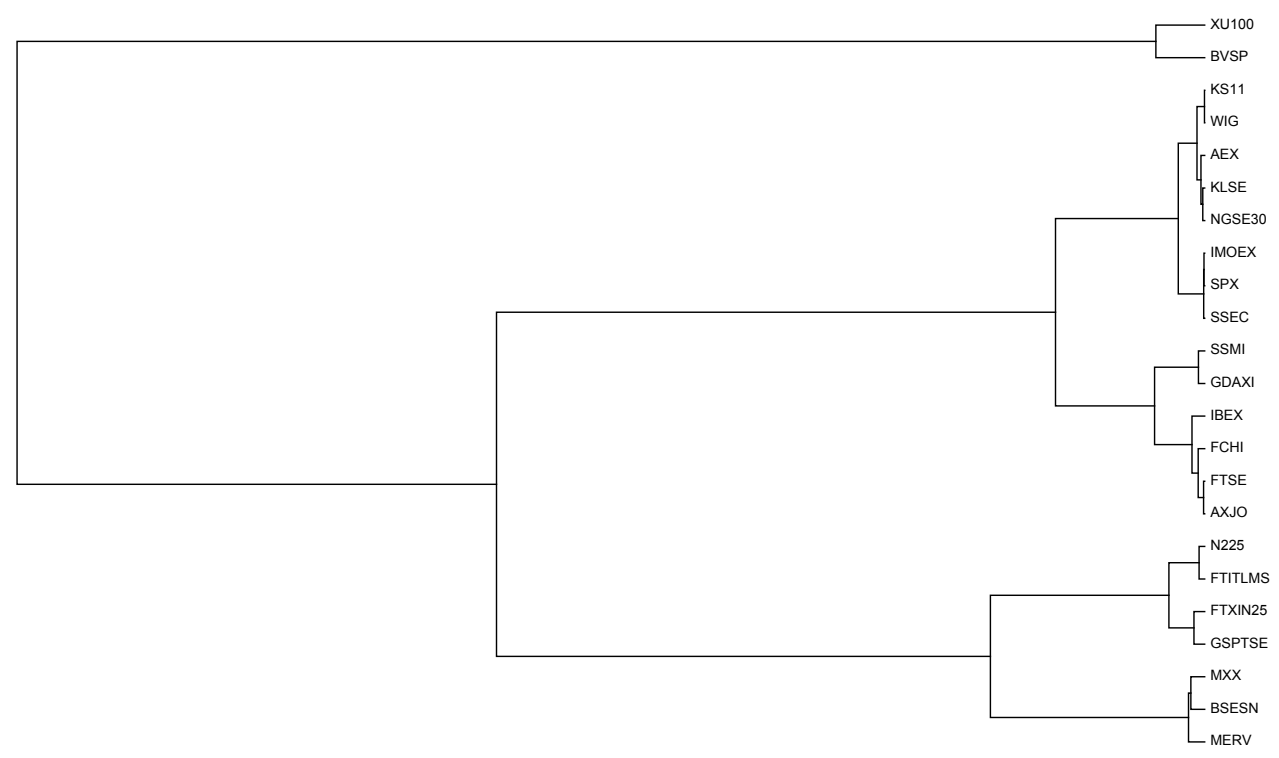

Figure 4. Minimum spanning tree of investigated stock markets during COVID-19

Table 3. Mean similarity measure, normalized tree length, characteristic path length of investigated stock markets before and during COVID-19

$\begin{array}{ll}\text { Before COVID-19 } & \text { During COVID-19 } \\ 1.37159 * 10^{7} & 1.82748 * 10^{6} \\ 2.28542 * 10^{6} & 287655 \\ 8.24901 & 8.36759\end{array}$

Mean similarity measure

Normalized tree length

8.24901

8.36759 
S\&P/BMV IPC (Mexico), BSE Sensex 30 (India) and S\&P Merval. Those clusters show us 21 financial markets involved. From Figure 3 it is seen that BOVESPA and BIST100 indices behave relatively similar to each other. However, they can not form a cluster due to not having enough (at least) stock markets for topological evaluation. When we consider During COVID-19 pandemic we realize a very interesting result. Surprisingly, all clusters remain same in Figure 4 as in Figure 3. This result yields us to analyze the difference between two situations by topological evaluation. In Table 4 and Table 5 we present the topology evaluation of all clusters for both situations. Regarding to Table 4 nodes in the third cluster has the smallest value so the stock markets in the third cluster are close to each other by comparing to other clusters before COVID-19 pandemic. Similarly, one can see that from Table 5 the stock exchanges in the Cluster 3 remain closest during COVID-19 pandemic. Further, the characteristic path lengths are investigated. The results from Table 4 indicate that Cluster 2 is the tightest and Cluster 3 is the loosest cluster. From Table 5 it can be said that both Cluster 1 and Cluster 2 become more compact, however surprisingly characteristic path length of Cluster 3 remains same.

Table 4. Mean similarity measure, normalized tree length, characteristic path length of clusters before COVID-19

$\begin{array}{llll} & \text { Cluster 1 } & \text { Cluster 2 } & \text { Cluster 3 } \\ \text { Mean similarity measure } & 6.41966 * 10^{6} & 1.57449 * 10^{6} & 489472 \\ \text { Normalized tree length } & 1.9511 * 10^{6} & 665269 & 171418 \\ \text { Characteristic path length } & 3.66667 & 3.13333 & 3.82143\end{array}$

Table 5. Mean similarity measure, normalized tree length, characteristic path length of clusters during COVID-19

$\begin{array}{llll} & \text { Cluster 1 } & \text { Cluster 2 } & \text { Cluster 3 } \\ \text { Mean similarity measure } & 791813 & 245927 & 95536.7 \\ \text { Normalized tree length } & 276200 & 90034.4 & 24990.5 \\ \text { Characteristic path length } & 3.38095 & 3.33333 & 3.82143\end{array}$

\section{Conclusion}

COVID-19 pandemic is an unpredicted problem for whole world in many different ways. This study aims to analyze the impact of such an unpredicted problem on stock markets network by an application of operational research with a quantitative and a mathematical approach. Here we grouped stock markets and investigated whether there is any switch between groups or not. In order to understand the behavior of investigated stock markets we consider only daily stock market returns. For comparison before COVID-19 and during COVID-19 investigated time series divided into two time frames. Then minimum spanning trees for both time frames are constructed. It 
is seen from the comparison of constructed minimum spanning trees that the central nodes for each time frame has changed. Before COVID-19 pandemic, SMI (Switzerland) and S\&P 500 (United States) were the most dominant indices, but during COVID-19 pandemic Nikkei 225 (Japan) and MOEX Russia (Russia) indices became a dominant position. There might be several reasons for that origin change such as political issues, actions and reactions of policy makers, news, and company news. All those subjects could be studied separately or together in various disciplines.

We obtained the dendrograms for each time frame by using the constructed minimum spanning trees. Then three clusters obtained from each dendrogram. Surprisingly, the obtained clusters for each time frame remain exactly same. Those clusters are as follows: Cluster 1: NSE 30 (Nigeria), FTSE Malaysia KLCI (Malaysia), AEX (Netherlands), WIG (Poland), KOSPI (South Korea), MOEX Russia (Russia), S\&P 500 (United States) and Shanghai (China). Cluster 2: IBEX 35 (Spain), SMI (Switzerland), DAX (Germany), FTSE 100 (United Kingdom), CAC 40 (France) and S\&P/ASX 200 (Australia). Cluster 3: Nikkei 225 (japan), FTSE Italia (Italy), FTSE China 50 (Hong Kong), S\&P/TSX (Canada), S\&P/BMV IPC (Mexico), BSE Sensex 30 (India) and S\&P Merval (Argentina). It can be seen from the findings that an unpredicted global factor like COVID-19 pandemic can not cause a switch between the groups of stock exchanges. There might be other factors like political issues, local economical crises, commodity prices. The factors which cause a change within the clusters left as an open problem and might be tested in future studies.

Presented results might be useful for the investors for global stock markets. For future studies findings of this study could be tested and compared by using classical correlation distance measures only if fixing an average start date for COVID-19. Also this study can be extended by defining stock market returns as a function of some other factors related or unrelated to COVID-19. For example daily confirmed cases and deaths, exchange rates, commodities such as gold and oil prices could be considered as variables. Also, interested researchers could extend and compare the findings of this paper by investigating the values for the post-COVID-19 pandemic.

\section{References}

[1] Akgüller, Ö. (2019). A threshold method for financial networks and geometric scattering of agents. Communications in Statistics: Case Studies, Data Analysis and Applications, 5, 3, 230-242.

[2] Akgüller, Ö., Balcı, M. A. (2018). Geodetic convex boundary curvatures of the communities in stock market networks. Physica A: Statistical Mechanics and its Applications, 505, 569-581.

[3] Al-Awadhi, A. M., Al-Saifi, K., Al-Awadhi, A., Alhamadi, S. (2020). Death and contagious infectious diseases: Impact of the COVID-19 virus on stock market returns. Journal of Behavioral and Experimental Finance, 100326. 
[4] Ashraf, B. N. (2020). Economic impact of government interventions during the COVID-19 pandemic: International evidence from financial markets. Journal of Behavioral and Experimental Finance, 27, 100371.

[5] Ashraf, B. N. (2020). Stock markets' reaction to COVID-19: cases or fatalities? Research in International Business and Finance, 101249.

[6] Baker, S. R., Bloom, N., Davis, S. J., Kost, K., Sammon, M., Viratyosin, T. (2020). The unprecedented stock market reaction to COVID-19. The Review of Asset Pricing Studies. https://doi.org/10.1093/rapstu/raaa008

[7] Balc1, M. A. (2018). Hierarchies in communities of Borsa Istanbul stock exchange. Hacettepe Journal of Mathematics and Statistics, 47, 4, 921-936.

[8] Balcı, M. A., Akgüller, Ö., Güzel, S. C. (2020). Hierarchies in communities of UK stock market from the perspective of Brexit. Journal of Applied Statistics, 1-19.

[9] Dashraath, P., et al. (2020). Coronavirus disease 2019 (COVID-19) pandemic and pregnancy. American journal of obstetrics and gynecology.

[10] Gates, B. (2020). Responding to Covid-19 -a once-in-a-century pandemic?. New England Journal of Medicine, 382, 18, 1677-1679.

[11] Goodell, J. W. (2020). COVID-19 and finance: Agendas for future research. Finance Research Letters, 101512.

[12] Guan, W. J., et al. (2020). Clinical characteristics of coronavirus disease 2019 in China. New England journal of medicine, 382, 18, 1708-1720.

[13] Hatipoğlu, V. F. (2017). Application of a New Quantitative Approach to Stock Markets: Minimum Spanning Tree. Alphanumeric Journal, 5, 1, 163-169.

[14] Holmes, et al. (2020). Multidisciplinary research priorities for the COVID-19 pandemic: a call for action for mental health science. The Lancet Psychiatry.

[15] Jang, W., Lee, J., Chang, W. (2011). Currency crises and the evolution of foreign exchange market: Evidence from minimum spanning tree, Physica A, 390, $707-718$.

[16] Kazemilari, M., Mohamadi, A., Mardani, A., Streimikis, J. (2019). Network topology of renewable energy companies: minimal spanning tree and sub-dominant ultrametric for the American stock. Technological and Economic Development of Economy, 25, 2, 168-187.

[17] Kwapien, J., Gworek, S., Drozdz, S. (2009). Structure and evolution of the foreign exchange networks, Acta Physica Polonica B, 40, 175-194.

[18] Li, B., Liao, Z. (2019). Finding changes in the foreign exchange market from the perspective of currency network. Physica A: Statistical Mechanics and its Applications, 545, 123727. 
[19] Liu, Z., Magal, P., Seydi, O., Webb, G. (2020). A COVID-19 epidemic model with latency period. Infectious Disease Modelling, 5, 323-337.

[20] Lyócsa, Š., Baumohl, E., Vỳrost, T., Molnár, P. (2020). Fear of the coronavirus and the stock markets. Finance research letters, 101735.

[21] Nguyen, Q., Nguyen, N. K. K., Nguyen, L. H. N. (2019). Dynamic topology and allometric scaling behavior on the Vietnamese stock market. Physica A: Statistical Mechanics and its Applications, 514, 235-243.

[22] Nguyen, Q., Nguyen, N. K. K. (2019). Composition of the first principal component of a stock index-A comparison between SP500 and VNIndex. Physica A: Statistical Mechanics and its Applications, 536, 120980.

[23] Nicola, M., et al. (2020). The socio-economic implications of the coronavirus pandemic (COVID-19): A review. International journal of surgery (London, England), 78, 185.

[24] Noraee, S., Bahrol Olom, H. (2020). Responding to Covid-19 -A Once-in-aCentury Pandemic?. Biological Science Promotion, 3, 6, 162-164.

[25] Petropoulos, F., Makridakis, S. (2020). Forecasting the novel coronavirus COVID-19. PloS one, 15, 3, e0231236.

[26] Pfefferbaum, B., North, C. S. (2020). Mental health and the Covid-19 pandemic. New England Journal of Medicine.

[27] Phan, D. H. B., Narayan, P. K. (2020). Country responses and the reaction of the stock market to COVID-19-A preliminary exposition. Emerging Markets Finance and Trade, 56, 10, 2138-2150.

[28] Roosa, K., et al. (2020). Real-time forecasts of the COVID-19 epidemic in China from February 5th to February 24th, 2020. Infectious Disease Modelling, 5, 256263.

[29] Saini, R., Kumar, P., Roy, P. P., Pal, U. (2019). Modeling Local and Global Behavior for Trajectory Classification using Graph Based Algorithm. Pattern Recognition Letters.

[30] Shakil, M. H., Munim, Z. H., Tasnia, M., Sarowar, S. (2020). COVID-19 and the environment: A critical review and research agenda. Science of the Total Environment, 141022.

[31] Topcu, M., Gulal, O. S. (2020). The impact of COVID-19 on emerging stock markets. Finance Research Letters, 101691.

[32] Wagner, A. F. (2020). What the stock market tells us about the post-COVID-19 world. Nature Human Behaviour, 4, 5, 440-440. 
[33] Wang, G. J., Xie, C., Han, F., Sun, B. (2012). Similarity measure and topology evolution of foreign exchange markets using dynamic time warping method: Evidence from minimal spanning tree. Physica A: Statistical Mechanics and its Applications, 391, 16, 4136-4146.

[34] Yue, P., Gizem Korkmaz, A., Zhou, H. (2020). Household financial decision making amidst the COVID-19 pandemic. Emerging Markets Finance and Trade, 56, $10,2363-2377$.

[35] Zou H., Yang J., (2019). Dynamic thresholding networks for schizophrenia diagnosis. Artificial intelligence in medicine, 96, 25-32.

Received 10.06.2020, Accepted 29.12.2020 